\title{
Comparison between anopheline mosquitoes (Diptera: Culicidae) caught using different methods in a malaria endemic area of Papua New Guinea
}

\author{
J.L.K. Hii ${ }^{*}$, T. Smith ${ }^{2}$, A. $\mathrm{Mai}^{3}$, E. Ibam $^{3}$ and M.P. Alpers ${ }^{3}$ \\ ${ }^{1}$ School of Public Health and Tropical Medicine, James Cook University, \\ Townsville, Q4810, Australia: ${ }^{2}$ Public Health and Epidemiology, Swiss \\ Tropical Institute, Socinstrasse 57, Postfach CH-4002, Basel, Switzerland: \\ ${ }^{3}$ Papua New Guinea Institute of Medical Research, Madang and Goroka, \\ Papua New Guinea
}

\begin{abstract}
The mosquito sampling efficiency of CDC (Centers for Disease Control) miniature light traps hung adjacent to mosquito nets, was compared with that of both indoor and outdoor human-bait collections in ten villages in the Wosera area of Papua New Guinea. The most frequently collected anopheline in the matched indoor and light trap samples was Anopheles koliensis Owen, followed by $A$. punctulatus Dönitz, A. karwari (James), A. farauti Laveran (sensu lato), A. longirostris Brug and A. bancroftii Giles. All species were much less frequent in the light traps than in landing catches. The hypothesis that the numbers of mosquitoes in light traps are proportional to human landing catches was examined using regression models that allowed for sampling error in both entomological measurements. Light traps under-sampled A. punctulatus and A. farauti s.l. at high densities. The models indicated that the ratio of light trap to landing catch females of $A$. koliensis and $A$. karwari increased with increasing mosquito density. Light trap catches of $A$. longirostris were proportional to indoor landing rates but when outdoor landing rates were high this species was under-sampled by light traps. Numbers of $A$. bancroftii in light traps were found to be proportional to those in outdoor landing catches, but were negatively related to those attempting to bite indoors. Circumsporozoite positivity rates for both Plasmodium falciparum Welch and P. vivax (Grassi \& Feletti) in A. punctulatus and A. farauti s.l. were significantly higher in light trap collections than in either indoor or outdoor landing catches, suggesting that light traps may selectively sample older mosquitoes of these species.
\end{abstract}

\section{Introduction}

Epidemiological studies of many vector-borne diseases are seriously hampered by the scarcity of objective sampling methods for mosquito vector populations. The collection of malaria mosquitoes landing on human 'baits' is considered

Address for correspondence: AusAID, HLG, GPO Box 887, Canberra, ACT 2601, Australia

Fax: +6126206 4870

E-mail: jeffrey_hii@ausaid.gov.au the most representative method for determining humanbiting activity since female mosquitoes are collected as they attempt to feed on the human collectors (WHO, 1975). However, the all-night collection method is labour intensive and unreliable because of variation in host attractiveness of human collectors (Lindsay et al., 1993; Knols et al., 1995). Such collections may also be unethical when the mosquito catchers are exposed to potentially infected mosquitoes.

Light traps set beside occupied bednets present an alternative to human biting catches and avoid the possible ethical problem that arises when mosquito collectors deliberately expose themselves to disease vectors (Lines et 
al., 1991). They also give more reproducible catches than standard human-biting collections, and hence, assuming that light traps can be calibrated against the standard, they provide a relatively easy way of obtaining precise estimates of the human biting rate.

Comparisons between light trap catches and biting/landing catches have given varying results, depending on the vectors concerned. In Tanzania, the catches of Anopheles gambiae s.l. Giles and Culex quinquefasciatus Say (Diptera: Culicidae) in light traps set beside occupied untreated bednets were proportional to those in the same week and village as human biting catches, and the age distribution of the mosquitoes caught by the two methods were similar (Lines et al., 1991). However, Mbogo et al. (1993) claimed that proportionality was not observed with Anopheles gambiae s.l. in Kenya. The statistical methodology used for these comparisons may account for some of this inconsistency (Smith, 1995).

Interspecific and geographic variation in anopheline behaviour may also be important. For instance, with Anopheles nuneztovari Gabaldon (Diptera: Culicidae) in Venezuela, the efficiency of light traps was lower than recorded in East Africa and the relationship between light trap catches and human biting less reliable (Rubio-Palis \& Curtis, 1992; Rubio-Palis, 1996). We now consider the relationships between numbers of anopheline mosquitoes caught in a study of the malaria vectors in an endemic area of Papua New Guinea (Hii et al., 1997) using three different methods: all night indoor landing catches, outdoor landing catches and standard Centers for Disease Control (CDC) (Atlanta, USA) miniature light traps (Sudia \& Chamberlain, 1962). To evaluate whether the methods sample the same fractions of the mosquito populations, and could hence be calibrated against each other, we therefore use a novel statistical method based on modelling the sampling variation with Poisson distributions.

\section{Materials and methods}

\section{Study area}

With informed consent and active cooperation of the villagers, this study was undertaken during 1991 to 1993 among ten villages (population 100-800 each) of the Wosera district $\left(143^{\circ} \mathrm{E} 52^{\prime} \mathrm{S}\right)$ of East Sepik Province, Papua New Guinea. The description of the Wosera area and the maps showing the location of the ten villages (Apusit, Kunjingini1, Nale-1, Nale-2, Kunjingini-2, Mul, Kamge, Kausagu, Nindigo, Nainda) are provided elsewhere (Hii et al., 1997).

\section{Sampling}

Landing collections and CDC light trap collections were performed concurrently with light traps for five consecutive nights per month commencing in June 1990 among eight villages and extending to another two villages in September (Hii et al., 1997). Depending on geographic size, each village was divided into five or six sectors; collectors rotated through the sections on different nights, and thus sampling was repeated in each section once a month, with one house from each section chosen each month at random, for replacement.

Mosquitoes landing on humans from $1800 \mathrm{~h}$ to $0600 \mathrm{~h}$ were caught by adult volunteers from the local population with feet and legs bared to the knees. Using aspirators aided by light from torches, the collectors captured mosquitoes that landed on them in search of a blood meal. Collectors worked in pairs for 6-h shifts. One pair began at $1800 \mathrm{~h}$ and the other at midnight. One individual of a pair of mosquito catchers worked inside the selected house, and the other outside the house.

Corresponding to each pair of landing catches, miniature CDC light traps were operated in four houses chosen to be as near to the landing collections as possible while not being visible from them. Each light trap, equipped with four zinccarbon type D batteries, a $100 \mathrm{~mA}$ incandescent bulb and a lid, was hung inside a bedroom where people slept. The inlet of the trap was set to $c .1 .8 \mathrm{~m}$ from the floor or ground and $20-100 \mathrm{~cm}$ from an insecticide-free mosquito net. At the time of the study, an insecticide-treatment bednet programme was not available in the Wosera area. The use of mosquito nets by inhabitants of those houses was monitored by the collectors. The present analyses consider light trap data only for houses in which all occupants were sleeping beneath nets. Traps were switched on at $2000 \mathrm{~h}$ local time, and collectors switched them off at $0600 \mathrm{~h}$, after tying the neck of the collecting bag.

\section{Circumsporozoite protein analysis}

Head/thorax portions or whole bodies of silica-dried female anophelines were tested by enzyme-linked immunosorbent assay (ELISA) using the 2A10, NSV3, 247K and 109-1794 (IgG3) monoclonal antibodies to detect circumsporozoite (CS) protein of Plasmodium falciparum (Welch), $P$. vivax (Grassi \& Feletti) (VK210 and VK247 variants) and $P$. malariae (Laveran) respectively (Wirtz et al., 1987, 1990, 1992; Collins et al., 1988).

\section{Statistical methods}

In general, the problem of evaluating agreement between different measurement techniques can be addressed using plots of differences between pairs of measurements against averages of the two methods (Altman \& Bland, 1983). This approach has been applied to logarithmically transformed mosquito collection data (Lines et al., 1991; Mbogo et al., 1993; Davis et al., 1995), but must be modified if it is to allow for zero counts of mosquitoes. The simple expedient of adding one to each count can be misleading (Smith, 1995), and we therefore used a regression based method.

Random sampling of a homogeneous population of mosquitoes would give a Poisson distribution of counts (in which the variance is equal to the mean). In addition to this random sampling variation, however, mosquito counts vary considerably as a result of differences in underlying densities and therefore the observed variance in recorded mosquito numbers is typically greater than the mean (overdispersion). One modelling approach is therefore to assume the counts to be distributed according to a negative binomial distribution. Negative binomial distributions have been extensively used to model densities of insects (see the review by Taylor, 1984), including mosquitoes (Nedelman, 1983).

The negative binomial can be parameterized as a gamma mixture of Poisson distributions (Venables \& Ripley, 1994), and we use this idea of a mixture of Poissons to model agreement between sampling methods. The recorded landing catches were assumed to be generated from an underlying distribution of densities (gamma or log normal), 
taking a distinct value, $\mathrm{E}\left(x_{i}\right)$, for each matched set, $i$, of one landing catch and four light traps. i.e.:

$$
\mathrm{E}\left(x_{i}\right) \sim \operatorname{Gamma}\left(\alpha_{0}, \alpha_{1}\right) \text { or } \log \left(\mathrm{E}\left(x_{i}\right)\right) \sim \operatorname{Normal}\left(\mu, \sigma^{2}\right)
$$

where $\alpha_{0}, \alpha_{1}, \mu$, and $\sigma^{2}$ are unknown parameters of the distributions of $\mathrm{E}\left(x_{i}\right)$.

We further propose that the sampling variation about $\mathrm{E}\left(x_{i}\right)$ was Poisson distributed, i.e.

$$
x_{i} \sim \operatorname{Poisson}\left(\mathrm{E}\left(x_{i}\right)\right)
$$

Where $\mathrm{E}\left(x_{i}\right)$ was taken to be gamma, the counts were therefore modelled as negative binomial. The assumption of a $\log$ normal distribution gave very similar values of $\mathrm{E}\left(x_{i}\right)$. In both cases the model handled zero counts naturally, with $x_{i}=$ 0 arising with probability $\exp \left(-\mathrm{E}\left(x_{i}\right)\right)$.

We fitted two distinct models for $y_{i, j}(j=1,2,3,4)$, the numbers of mosquitoes caught in the light traps corresponding to landing catch $i$

(i) A model assuming proportionality, i.e.

$$
\mathrm{E}\left(y_{i}\right)=\beta_{0} \mathrm{E}\left(x_{i}\right)
$$

(ii) A model allowing for a curvilinear relationship between the number of mosquitoes caught using the different methods.

$$
\mathrm{E}\left(y_{i}\right)=\beta_{1} \mathrm{E}\left(x_{i}\right)^{\beta_{2}}
$$

In both cases, we assumed:

$$
y_{i, j} \sim \text { Poisson }\left(\mathrm{E}\left(y_{i}\right)\right)
$$

It is not obvious how to fit these models by maximum likelihood, because the likelihood for $y_{i j}$ and $x_{i}$ includes terms in the unknown $\mathrm{E}\left(x_{i}\right)$. However, the independence of $y_{i, j}$ and $x$ conditional on $\mathrm{E}\left(x_{i}\right)$, means that the posterior distributions of the parameters can be sampled using a (Bayesian) Gibbs Sampling approach. In separate models, the parameters of either (3) or (4) above, and of (1) were thus estimated for each species of Anopheles, and both indoor and outdoor landing catches. Estimation used the program BUGS (Bayesian Updating using Gibbs Sampling), with non-informative priors for $\beta_{0}, \beta_{1}$ and $\beta_{2}, \alpha_{0}, \alpha_{1}, \mu$ and $\sigma^{2}$ (Thomas et al., 1992).

Agreement between the different sampling methods was assessed:

(i) by calculation of Deviance statistics to examine the fit of the models. These were calculated as:

$$
\mathrm{d}_{x}=2 \sum_{\mathrm{i}}\left[x_{\mathrm{i}} \ln \left(\left(x_{\mathrm{i}}+0.5\right) / \mathrm{E}\left(x_{\mathrm{i}}+0.5\right)\right)-\left(x_{\mathrm{i}}-\mathrm{E}\left(x_{\mathrm{i}}\right)\right)\right]
$$

and

$$
\mathrm{d}_{y}=2 \sum_{\mathrm{i}, \mathrm{j}}\left[y_{\mathrm{ij}} \ln \left(\left(y_{\mathrm{i}, \mathrm{j}}+0.5\right) / \mathrm{E}\left(y_{\mathrm{i}}+0.5\right)\right)-\left(y_{\mathrm{i}, \mathrm{j}}-\mathrm{E}\left(y_{\mathrm{i}}\right)\right)\right]
$$

where the sum $\left(d_{x}+d_{y}\right)$ should be approximately equal to the residual degrees of freedom if the fit of the lines is good and the sampling error is approximately Poisson, as assumed. For the calibration of the methods against each other to be meaningful, there should not be very substantial variation about the regression lines, i.e. the deviance statistic should be small.

(ii) by comparing the two different regression lines to see if the proportionality assumption holds. If the different methods are sampling the same fraction of the mosquito population then the fitted line for model (ii) should be close to that for model (i). Equivalently, the $95 \%$ credible intervals (Bayesian equivalent of confidence intervals) for $\beta_{2}$ should overlap with unity.

\section{Results}

The spatial and temporal distributions of anopheline mosquitoes (Diptera: Culicidae) in the area, and their taxonomy, have been discussed elsewhere (Hii et al., 1997). Table 1 gives total numbers caught in the matched indoor and CDC traps. The most frequent species was Anopheles koliensis Owen. Anopheles punctulatus Dönitz was also frequent and a further four species, Anopheles karwari (James), Anopheles farauti Laveran (sensu lato), Anopheles bancroftii Giles and Anopheles longirostris Brug occurred relatively infrequently.

The distribution of Anopheles species sampled by light traps varied between villages. This pattern parallels that of the landing catches which we have presented elsewhere (compare table 2 with the data of Hii et al., 1997).

The numbers of mosquitoes caught in outdoor landing catches were similar to those landing on indoor mosquito collectors, but all Anopheles species except A. koliensis were much less frequent in the light traps than in either landing collection (table 1). This is illustrated both by the ratios of the mean numbers caught for the two trapping methods (table 1) and by the best estimates of the constants of proportionality derived from model (i) (table 3), which are, in all cases, similar.

The relatively low numbers of mosquitoes caught in the light traps do not on their own, however, invalidate the use of this sampling technique for estimating biting densities. The crucial criterion is that of whether the catching methods give numbers of mosquitoes which are in proportion to each

\begin{tabular}{|c|c|c|c|c|c|c|c|c|}
\hline & \multicolumn{2}{|c|}{ Indoor landing } & \multicolumn{2}{|c|}{ Outdoor landing } & \multicolumn{2}{|c|}{ Light traps } & \multicolumn{2}{|c|}{ Ratios of light trap: landing } \\
\hline & Total & $\begin{array}{c}\text { Rate } \\
\left(\text { person }^{-1} n^{-1}\right)\end{array}$ & Total & $\begin{array}{c}\text { Rate } \\
\left.\text { (person }^{-1} n^{-1}\right)\end{array}$ & Total & $\begin{array}{c}\text { Rate } \\
\left(\operatorname{trap}^{-1} \mathrm{n}^{-1}\right)\end{array}$ & Indoors & Outdoors \\
\hline Anopheles punctulatus & 5453 & 11.5 & 6007 & 12.6 & 2031 & 1.11 & 0.1 & 0.09 \\
\hline A. koliensis & 9347 & 19.6 & 9777 & 20.5 & 10346 & 5.68 & 0.29 & 0.28 \\
\hline A. karwari & 3007 & 6.32 & 2981 & 6.27 & 1595 & 0.88 & 0.14 & 0.14 \\
\hline A. farauti & 603 & 1.27 & 673 & 1.41 & 174 & 0.1 & 0.08 & 0.07 \\
\hline A. bancroftii & 111 & 0.23 & 158 & 0.33 & 87 & 0.05 & 0.2 & 0.14 \\
\hline A. longirostris & 197 & 0.41 & 341 & 0.72 & 78 & 0.04 & 0.1 & 0.06 \\
\hline No. of collections & 476 & & 476 & & 1822 & & & \\
\hline
\end{tabular}
other. If this condition is not fulfilled, then it is unlikely that they are sampling the same fraction of the mosquito population.

In the comparison of indoor landing with light trap collections (fig. 1) with the exception of A. longirostris

Table 1. Numbers of anopheline mosquitoes caught in matched landing and light trap catches.

Includes only CDC traps in houses where all sleepers used bednets, and matched landing catches.

$\mathrm{n}=$ night. 
Table 2. Numbers of anopheline mosquitoes caught in light traps, by village.

\begin{tabular}{lccccccc}
\hline Village & $\begin{array}{c}\text { No. of } \\
\text { trap nights }\end{array}$ & \multicolumn{5}{c}{ Williams' mean ${ }^{1}$ number of mosquitoes per trap-night } \\
\cline { 3 - 7 } & & A. punctulatus & A. koliensis & A. karwari & A. farauti & A. bancroftii & A. longirostris \\
\hline Apusit & 323 & 0.69 & 1.26 & 0.05 & 0.04 & 0.03 & 0.03 \\
Kamge & 99 & 0.22 & 0.28 & 0.01 & 0.03 & 0.11 & 0.00 \\
Kunjingini-1 & 256 & 0.63 & 1.57 & 0.06 & 0.06 & 0.02 & 0.04 \\
Kunjingini-2 & 191 & 1.00 & 2.15 & 0.02 & 0.01 & 0.01 & 0.03 \\
Mul & 296 & 0.54 & 2.04 & 0.06 & 0.02 & 0.01 & 0.03 \\
Nale-1 & 263 & 0.27 & 2.24 & 0.10 & 0.69 & 0.05 & 0.08 \\
Nale-2 & 144 & 0.56 & 3.14 & 0.13 & 0.92 & 0.03 & 0.01 \\
Kausagu & 93 & 0.27 & 0.22 & 0.03 & 0.02 & 0.00 & 0.01 \\
Nainda & 39 & 0.14 & 1.31 & 0.05 & 0.04 & 0.02 & 0.04 \\
Nindigo & 118 & 0.19 & 0.79 & 0.01 & 0.01 & 0.01 & 0.01 \\
\hline
\end{tabular}

${ }^{1}$ Williams' mean $=\exp \left[\left(\bullet \ln \left(\mathrm{y}_{\mathrm{ij}}+1\right)\right) / \mathrm{n}\right]-1$ where $\mathrm{n}$ is the number of trap-nights.

(indoor landing) and A. bancroftii (outdoor landing), the 95\% interval estimates for the exponents ( $\beta_{2}$ from model (ii)) did not overlap with unity (table 3 ). This indicates that the numbers of mosquitoes caught by the different methods are not proportional. However, the deviation from proportionality was quite small in most cases. The exceptions were $A$. farauti s.l., which showed a strongly curvilinear relationship (fig. 1) and A. bancroftii, where light trap densities decreased with increase in indoor landing catches. In all species except A. bancroftii, the parameter estimates for the models fitted to outdoor landing data were similar to those for indoor landing. In A. bancroftii, the curve fitted by Model (ii) to indoor landing data indicated a decrease in light trap densities with increasing landing rates, whilst outdoor landing densities for A. bancroftii were proportional to light trap densities.

The scatters of light trap catches against the matched indoor landing catches suggest that there is considerable variation about the fitted relationships between the two trapping methods (fig. 1). In A. punctulatus, and especially in A. koliensis, the dispersion was in excess of that expected in the Poisson model, indicating that there is substantial scatter in excess of sampling variation (table 3 ). The deviance statistics for the four rarer Anopheles species were all small, reflecting the sparseness of the data. When the data are sparse, then the deviance is a poor measure of the fit of the model. Hence for these species, no conclusions can be drawn about the degree of dispersion about the fitted lines.

Table 4 shows the circumsporozoite protein (CS) positivity rates estimated separately for each Anopheles species and for each of the three methods of sampling. For all three of the Plasmodium species found in the area (table 4), and for both the VK210 and VK247 variants of P. vivax, CSpositive mosquitoes were detected in all six anopheline species. However CS proteins of P. malariae occurred only sporadically, and CS positivity rates were uniformly low in $A$. farauti s.l. and in whole bodies of $A$. longirostris and $A$. bancroftii (table 4). Anopheles punctulatus and A. koliensis had the highest CS positivity rates, both for $P$. falciparum and for P. vivax (table 4). Anopheles koliensis, A. farauti s.l. and A. longirostris were also the anophelines with the highest human blood indexes ( $\geq 0.84)$ (Hii et al., 1997).

In A. punctulatus and A. farauti s.l., the P. falciparum CS positivity rate was significantly higher in light trap

Table 3. Parameter estimates from hierarchical models (95\% intervals).

\begin{tabular}{lllllll}
\hline $\begin{array}{l}\text { Anopheline } \\
\text { species }\end{array}$ & A. punctulatus & A. koliensis & A. karwari & A. farauti s.l. & A. bancroftii & A. longirostris \\
\hline Indoor landinga & & & & & \\
$\beta_{0}$ (Model (i)) & $0.10(0.09,0.11)$ & $0.28(0.27,0.29)$ & $0.12(0.11,0.13)$ & $0.074(0.059,0.090)$ & $0.20(0.15,0.27)$ & $0.12(0.08,0.15)$ \\
$\beta_{1}$ (Model (ii)) & $0.22(0.19,0.25)$ & $0.10(0.095,0.12)$ & $0.028(0.018,0.039)$ & $0.10(0.082,0.127)$ & $0.002(0.0007,0.005)$ & $0.12(0.08,0.16)$ \\
$\beta_{2}$ (Model (ii)) & $0.76(0.71,0.80)$ & $1.27(1.22,1.29)$ & $1.36(1.28,1.45)$ & $0.54(0.37,0.70)$ & $-0.915(-1.27,-0.68)$ & $0.83(0.58,1.05)$ \\
Deviance (Model (ii)) & 2959 & 9871 & 589 & 389 & 154 & 298 \\
Outdoor landingb & & & & & \\
$\beta_{0}$ (Model (i)) & $0.089(0.084,0.094)$ & $0.27(0.26,0.28)$ & $0.12(0.11,0.13)$ & $0.063(0.050,0.077)$ & $0.15(0.11,0.20)$ & $0.065(0.047,0.087)$ \\
$\beta_{1}$ (Model (ii)) & $0.26(0.22,0.30)$ & $0.16(0.13,0.18)$ & $0.015(0.009,0.022)$ & $0.095(0.076,0.116)$ & $0.15(0.10,0.21)$ & $0.081(0.057,0.109)$ \\
$\beta_{2}$ (Model (ii)) & $0.68(0.64,0.72)$ & $1.14(1.10,1.18)$ & $1.51(1.42,1.61)$ & $0.44(0.28,0.60)$ & $1.07(0.64,1.55)$ & $0.75(0.56,0.96)$ \\
Deviance (Model (ii)) & 2986 & 10600 & 1530 & 395 & 267 & 235 \\
\hline
\end{tabular}

Deviance on 1725 degees of freedom.

a Comparison of numbers caught in light traps with numbers of indoor landing mosquitoes.

$\mathrm{b}$ Comparison of numbers caught in light traps with numbers of outdoor landing mosquitoes.

Model (i): $\quad \mathrm{E}\left(y_{i}\right)=\beta_{0} \mathrm{E}\left(x_{i}\right)$

Model (ii): $\quad \mathrm{E}\left(y_{i}\right)=\beta_{1} \mathrm{E}\left(x_{i}\right)^{\beta_{2}}$

Parameter estimates quoted are those from models with $\mathrm{E}\left(x_{i}\right)$ from $\log$ normal distributions. Models with $\mathrm{E}\left(x_{i}\right)$ from gamma distributions gave very similar results. 

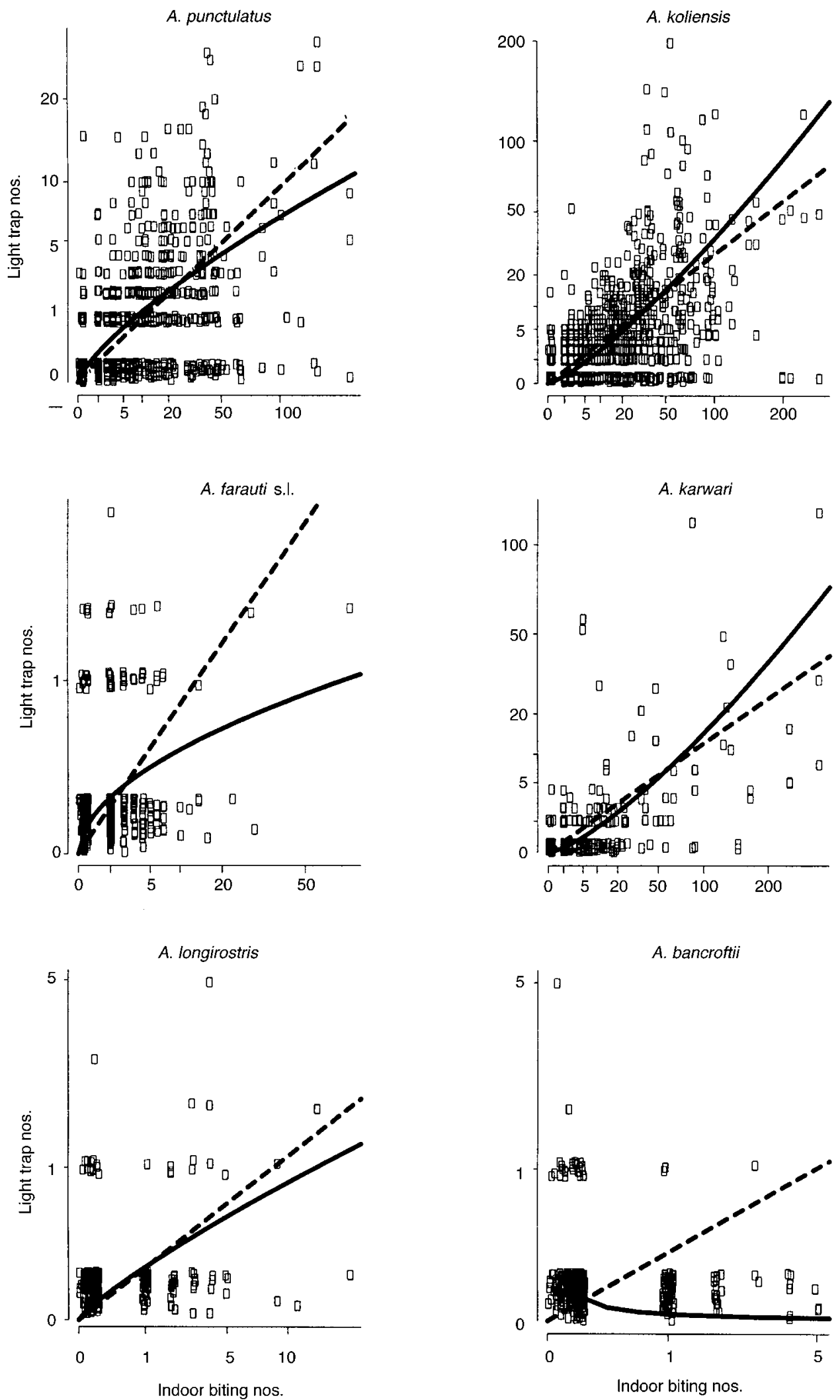

Fig. 1. Plots of numbers of anopheline mosquitoes caught in 1250 CDC light trap collections, against those caught in 476 indoor landing catches matched with the light trap collections. (A small amount of noise has been added to the data to distinguish superimposed points). Dashed line, fitted line from model (i); continuous line, fitted line from model (ii). 
Table 4. Numbers of anopheline mosquitoes (percentages) positive for malarial circumsporozoite protein.

\begin{tabular}{|c|c|c|c|c|c|c|}
\hline & $\begin{array}{c}\text { Anopheles } \\
\text { punctulatus }\end{array}$ & $\begin{array}{c}\text { A. } \\
\text { koliensis }\end{array}$ & $\begin{array}{c}A . \\
\text { karwari }\end{array}$ & $\begin{array}{c}A . \\
\text { farauti s.l. }\end{array}$ & $\begin{array}{c}A . \\
\text { bancroftii }\end{array}$ & $\begin{array}{c}A . \\
\text { longirostris }\end{array}$ \\
\hline \multicolumn{7}{|l|}{ Indoor landing } \\
\hline Total tested & 15999 & 14815 & 5381 & 1571 & 217 & 896 \\
\hline Plasmodium falciparum & $107(0.67)$ & $101(0.68)$ & $16(0.30)$ & $2(0.13)$ & $0(0.00)$ & $1(0.11)^{*}$ \\
\hline P. malariae & $12(0.08)$ & $8(0.05)$ & $2(0.04)$ & $1(0.07)$ & $0(0.00)$ & $1(0.11)^{*}$ \\
\hline P. vivax (VK210) & $17(0.11)$ & $8(0.05)$ & $1(0.02)$ & $0(0.00)$ & $0(0.00)$ & $1(0.11)^{*}$ \\
\hline P. vivax (VK247) & $28(0.18)$ & $32(0.22)$ & $3(0.06)$ & $0(0.00)$ & $0(0.00)$ & $1(0.11)^{*}$ \\
\hline P. vivax (total) & $45(0.28)$ & $40(0.26)$ & $4(0.07)$ & $0(0.00)$ & $0(0.00)$ & $2(0.22)^{*}$ \\
\hline \multicolumn{7}{|l|}{ Outdoor landing } \\
\hline Total tested & 18181 & 15328 & 5467 & 1701 & 266 & 1188 \\
\hline P. falciparum & $132(0.75)$ & $126(0.82)$ & $19(0.35)$ & $0(0.00)$ & $2(0.75)^{*}$ & $1(0.08)^{*}$ \\
\hline P. malariae & $17(0.09)$ & $10(0.07)$ & $3(0.05)$ & $3(0.18)$ & $1(0.38)^{*}$ & $0(0.00)$ \\
\hline P. vivax (VK210) & $13(0.07)$ & $23(0.15)$ & $3(0.05)$ & $1(0.06)$ & $1(0.38)^{*}$ & $0(0.00)$ \\
\hline P. vivax (VK247) & $32(0.18)$ & $41(0.27)$ & $3(0.05)$ & $1(0.06)$ & $1(0.38)^{*}$ & $0(0.00)$ \\
\hline P. vivax (total) & $45(0.25)$ & $64(0.42)$ & $6(0.11)$ & $2(0.12)$ & $2(0.75)$ & $0(0.00)$ \\
\hline \multicolumn{7}{|l|}{ Light traps } \\
\hline Total tested & 5358 & 13623 & 2286 & 379 & 159 & 181 \\
\hline P. falciparum & 61(1.14) & $113(0.83)$ & $7(0.31)$ & $3(0.79)$ & $0(0.00)$ & $0(0.00)$ \\
\hline P. malariae & $5(0.09)$ & $19(0.14)$ & $0(0.00)$ & $1(0.26)$ & $0(0.00)$ & $0(0.00)$ \\
\hline P. vivax (VK210) & $17(0.32)$ & $19(0.14)$ & $4(0.17)$ & $0(0.00)$ & $0(0.00)$ & $0(0.00)$ \\
\hline P. vivax (VK247) & $15(0.29)$ & $37(0.28)$ & $3(0.14)$ & $0(0.00)$ & $0(0.00)$ & $0(0.00)$ \\
\hline P. vivax (total) & $32(0.60)$ & $56(0.41)$ & $7(0.31)$ & $0(0.00)$ & $0(0.00)$ & $0(0.00)$ \\
\hline
\end{tabular}

* Sporozoite-positives found in whole bodies only.

collections than in either indoor or outdoor landing catches (A. punctulatus: Likelihood ratio $\chi^{2} 2$ d.f., $10.9, P=0.004 ; A$. farauti s.l.: Likelihood ratio $\chi^{2}, 2$ d.f., $\left.10.3, P=0.004\right)$. In $A$. punctulatus, the $P$. vivax CS positivity rate was also higher in light traps (Likelihood ratio $\chi^{2}, 2$ d.f., $14.1, P=0.004$ ). This pattern was not seen in $A$. koliensis. In $A$. bancroftii, CS proteins were only found in whole bodies of outdoor landing mosquitoes. In the other three anopheline species, only small numbers with CS proteins were detected and there were no clear relationships between CS positivity rates and trapping methods.

\section{Discussion}

We found that in the Wosera area of Papua New Guinea, light traps caught far fewer anophelines than does a human bait operating for an equivalent time period. At low mosquito densities, light traps placed in rooms where all potential human hosts were sleeping under mosquito nets, caught on average only about $10 \%$ of human landing catches of A. punctulatus and 29\% of A. koliensis. Minor vectors of malaria were also caught only at low densities in light traps. This confirms the observation of Bryan (1986) that CDC light traps do not catch many A. punctulatus complex mosquitoes in the Sepik area and was in agreement with Charlwood's et al. (1986a) comparative study of A. punctulatus complex in Papua New Guinea.

Other studies of a range of species in Cuba (Marquetti et al., 1992), Sabah (Hii et al., 1986), central Africa (Carnevale, 1973, 1974), east Africa (Lines et al., 1991; Mbogo et al., 1993; Githeko et al., 1994) and Venezuela (Rubio-Palis \& Curtis, 1992; Rubio-Palis, 1996) have also found that light traps catch fewer anophelines than human bait, although the relative efficiency of the two methods can vary considerably. Of 11 species caught in Western Venezuela, only Anopheles neomaculipalpus Curry was caught at similar rates in light traps and on human bait (Rubio-Palis, 1996). In contrast, five out of ten species in Peninsular Malaysia were caught at a higher rate in light traps (Parsons et al., 1974). Whilst a study of Anopheles fluviatilis James in India found that the relative trapping efficiencies varied by habitat (Gunasekaran et al., 1994), in Tanzania, the total number of anophelines captured overnight by light traps (hung beside a bednet in use) was 1.23 times the number of anophelines captured by humanbait collections (Davis et al., 1995). This relationship was not affected significantly by changes in the mosquito density, order of trapping method, date of sampling, or number of household occupants (Davis et al., 1995). However, higher catch rates were observed with the genetically-distinct West African populations of A. gambiae s.l. and Anopheles funestus Giles; their relative contribution to the probability of these vectors being captured was similar (about 2.5- and 3-fold increase for the light and net, respectively) and additive (Costantini et al., 1998).

Although the trapping efficiency of the light traps in Wosera was low, the traps would nevertheless be useful in quantifying vector populations if the numbers caught could be calibrated against landing densities. However, the plot of numbers of $A$. punctulatus caught in light traps against numbers in landing catches (fig. 1) indicated a wide degree of scatter with much greater dispersion than that expected if sampling error was Poisson distributed. There was even more scatter in the case of $A$. koliensis. It follows that although predictions of biting rates could be made from light trap catches using either model (i) or model (ii), these would be very imprecise. Such extra-Poisson variation in sampling error could arise if mosquitoes arrive at a house in groups: variation between species in the degree of overdispersion could easily reflect differences in the degree to which the mosquitoes cluster in this way.

To assess the calibration it was important to use statistical models that were not biased by the high frequency of zero counts in the data. For the less frequent species though, even this approach was less than optimal, because we could not 
interpret the deviance statistics in the presence of very large numbers of zero counts. The small sizes of the datasets for $A$. farauti s.l., A. longirostris and A. bancroftii in any case lead to very imprecise regression coefficients so that for these species any predictions would be very uncertain even without clustering.

Ideally, we would also hope to find that numbers of mosquitoes caught in light traps are proportional to those caught landing, as was found by Lines et al. (1991) for A. gambiae s.l. in Tanzania. Our regression analysis implied that light traps under-sampled $A$. punctulatus and $A$. farauti s.l. at high densities $\left(\beta_{2}<1\right)$. In contrast, for $A$. koliensis and $A$. karwari, the best fitting regression from model (ii) indicated that the ratio of light trap to landing catch mosquitoes increased with increasing mosquito density $\left(\beta_{2}>1\right)$. Only in the case of $A$. longirostris did the interval estimate for the exponent, $\beta_{2}$, overlap with 1 , indicating that the model of proportionality may be applicable over the whole range of mosquito densities in this species. However, when outdoor landing rates of $A$. longirostris were high, this species was under-sampled by light traps. Conversely, numbers of $A$ bancroftii caught in light traps were found to be proportional to those in outdoor landing catches, but were negatively related to the number attempting to bite indoors. The lack of proportionality in most of these analyses suggests that in any case, different fractions of the mosquito population were being sampled by the two different techniques, and thus that the light trap is a poor tool for estimating human biting densities of these species.

The higher sporozoite rates in light traps than landing catches of A. punctulatus and A. farauti s.l. suggest that the light traps were sampling a larger proportion of older or gravid mosquitoes of these species than the landing catches. Similar results have been obtained for some Afrotropical species. For example, parous rates in Anopheles nili Theobald were significantly greater in light traps (Carnevale \& Pont, 1973) and Mbogo et al. (1993) found higher P. falciparum sporozoite rates in A. gambiae s.l. from light traps than in those caught on human bait in contrast with Lines et al. (1991) who found similar age structures of A. gambiae in light trap and landing catches. In coastal Tanzania, Davis et al. (1995) found good agreement between light trap and nightlanding methods, which both showed that the P. falciparum sporozoite rate among anophelines captured by light trap was twice that of human-bait captured anophelines. These data are compatible with the 2.3-fold higher sporozoite rate among light trap collected anophelines in Kilifi, Kenya (Mbogo et al., 1993). Mbogo et al. (1993) argued that the mosquitoes caught in light traps may have been older than those on human bait because light traps can attract indoor resting mosquitoes, which will, on average, be older than those which are host-seeking. On the other hand, Davis et al. (1995) found that it was more likely that gravid mosquitoes in coastal Tanzania, with their higher sporozoite rates, were attracted to the light-source and captured while attempting to exit the house during the ovipositional flight (Service, 1970). Either of these age biases might have operated in our study. Anopheles punctulatus is probably the most endophilic of the Papua New Guinean species (Charlwood et al., 1986b), whilst the species of $A$. farauti s.l. found in Wosera has not yet been extensively studied. The differences are unlikely to be a consequence of differences in trap location (Gibb et al., 1988) because of the matching of locations of light traps and human bait.
In other studies, anophelines caught in light traps have been younger than those from landing catches. Species in which this trend has been found include A. nuneztovari (Rubio-Palis, 1996), A. gambiae (Carnevale \& Pont, 1973; Shidrawi et al., 1973) and Anopheles arabiensis Patton (Githeko et al., 1994). No difference in parous rates were found for A. gambiae s.l. by Lines et al. (1991), A. funestus (Githeko et al., 1994) or A. fluviatilis (Gunasekaran et al., 1994); however, the parous rate of unfed stage I or II females of Anopheles subpictus Grassi collected by light trap (blacklight fluorescent lamps) was significantly higher than that of cattle-bait samples (Mogi et al., 1995). It may be that the absence of sporozoites in those $A$. bancroftii caught indoors in our study indicates that only young mosquitoes of this species enter houses or the existence of cryptic species.

It may be that the discordance between sampling methods could be reduced by careful standardization of sampling methods. We did not standardize the light trap position in relation to the sleeper occupying the bednet, and trapping efficiency of light traps can be affected by nonrandom mosquito behaviour around bednets. In the raised feet position, light traps significantly sampled more $A$. gambiae in Tanzania than other trap positions, with significantly older mosquitoes caught near the top of the net than at the level of the host (Mboera et al., 1998). However, our overall conclusion must be that light traps cannot be calibrated to give reliable estimates of rates of human biting by anophelines in Papua New Guinea.

\section{Acknowledgements}

We are grateful to field teams who tirelessly collected and sorted light trap and landing catches over the years; to $\mathrm{M}$. Packer, E. Renyard, R. Paru, H. Dagoro, M. Lagog, M. Baea for technical and logistical support and to R.A. Wirtz for providing sporozoite monoclonal antibodies through the USAID grant. Drs J.D. Charlwood, J. Lines, M. Zalucki, S. Ritchie and two referees made valuable comments on an earlier version of the manuscript. We would particularly like to thank Dr N Alexander for useful and constructive discussions. Research clearance for this research was obtained from and approved by the Medical Research Advisory Committee of Papua New Guinea. This study was part of the Malaria Vaccine Epidemiology and Evaluation Project funded by the United States Agency for International Development, No. 9365967.89. Data analysis was partly supported by Swiss National Science Foundation grant 3243427.95 .

\section{References}

Altman, D.G. \& Bland, J.M. (1983) Measurement in medicine: the analysis of method comparison studies. The Statistician 32, 307-317.

Bryan, J.H. (1986) Vectors of Wuchereria bancrofti in the Sepik province of Papua New Guinea. Transactions of the Royal Society of Tropical Medicine and Hygiene 80, 123-131.

Carnevale, P. (1974) Comparaison de trois methodes de capture pour d'chantillonnage d'une population d'Anopheles nili. Cahiers ORSTOM Series Entomologie Médicale et Parasitologie 122, 135-144.

Carnevale, P. \& Le Pont, F. (1973) Epidemiologie du paludisme humain en Republique Populaire du Congo. 
II. Utilisation des piiges lumineux CDC comme moyen $\mathrm{d}^{\prime}$ chantillonnage des populations anopheliennes. Cahiers ORSTOM Series Entomologie Médicale et Parasitologie 114, 263-270.

Charlwood, J.D., Paru, R. \& Dagoro, H. (1986a) A new light-bed net trap to sample anopheline vectors of malaria in Papua New Guinea. Bulletin of the Society for Vector Ecologists 11, $1-3$.

Charlwood, J.D., Graves, P.M. \& Alpers, M.P. (1986b) The ecology of the Anopheles punctulatus group of mosquitoes from Papua New Guinea: a review of recent work. Papua New Guinea Medical Journal 29, 19-26.

Collins, F.H., Procell, P.M, Campbell, G.H. \& Collins W.E. (1988) Monoclonal antibody-based enzyme-linked immunosorbent assay (ELISA) for detection of Plasmodium malariae sporozoites in mosquitoes. American Journal of Tropical Medicine and Hygiene 38, 87-91.

Costantini, C., Sagnon, N.F., Sanogo, E., Merzagora, L. \& Coluzzi, M. (1998) Relationship to human biting collections and influence of light and bednet in CDC light trap catches of West African malaria vectors. Bulletin of Entomological Research 88, 503-511.

Curtis, C.F. (1992) Personal protection methods against vectors of disease. Reviews of Medical Entomology 80, 543-553.

Davis, J.R., Hall, T., Chee, E.M., Majala, A., Minjas, J. \& Shiff, C.J. (1995) Comparison of sampling anopheline mosquitoes by light trap and human-bait collections indoors at Bagamoyo, Tanzania. Medical and Veterinary Entomology 9, 249-255.

Gibb, P.A., Anderson, T.J.C. \& Dye, C. (1988) Are nulliparous sandflies light-shy? Transactions of the Royal Society of Tropical Medicine and Hygiene 82, 342-343.

Githeko, A.K., Service, M.W., Mbogo, C.M., Atieli, F.A. \& Juma, F.O. (1994) Sampling Anopheles arabiensis, A. gambiae sensu lato and A. funestus (Diptera: Culicidae) with CDC light traps near a rice irrigation area and a sugarcane belt in western Kenya. Bulletin of Entomological Research 84, 319-324.

Gunasekaran, K., Jambulingam, P., Sadanandane, C., Sahu, S.S. \& Das, P.K. (1994) Reliability of light trap sampling for Anopheles fluviatilis, a vector of malaria. Acta Tropica 58, $1-11$.

Hii, J., Chin, K.F., Macdonald, M. \& Vun, Y.S. (1986) The use of CDC light traps for malariometric entomology surveys in Sabah, Malaysia. Tropical Biomedicine 3, 39-48.

Hii, J.L.K., Smith, T., Mai, A., Mellor, S., Lewis, D., Alexander, N. \& Alpers, M.P. (1997) Spatial and temporal variation in abundance of Anopheles (Diptera: Culicidae) in a malaria endemic area in Papua New Guinea. Journal of Medical Entomology 34, 193-205.

Knols, J.G., de Jong, R. \& Takken, W. (1995) Differential attractiveness of isolated humans to mosquitoes in Tanzania. Transactions of the Royal Society of Tropical Medicine and Hygiene 89, 604-606.

Lindsay, S.W., Adiamah, J.H., Miller, J., Pleass, R.J. \& Armstrong, J.R.M. (1993) Variation in attractiveness of human subjects to malaria mosquitoes (Diptera: Culicidae). Journal of Medical Entomology 30, 368-373.

Lines, J.D., Curtis, C.F., Wilkes, T.J. \& Njunwa, K.J. (1991) Monitoring human-biting mosquitoes (Diptera: Culicidae) in Tanzania with light traps hung beside mosquito nets. Bulletin of Entomological Research 81,77-84.

Marquetti, M. del C., Navarro, A., Bisset, J. \& Garcia, F.A. (1992) Comparison of three catching methods for collecting anopheline mosquitoes. Memorias do Instituto Oswaldo Cruz $87,457-458$

Mboera, L.E.G., Kihonda, J., Braks, M.A.H. \& Knols, B.G.J. (1998) Short report: Influence of Centers for Disease Control light trap position, relative to a human-baited bed net, on catches of Anopheles gambiae and Culex quinquefasciatus in Tanzania. American Journal of Tropical Medicine and Hygiene 59, 595-596.

Mbogo, C.N.M., Glass, G.E., Forster, D., Kabiru, E.W., Githure, J.I., Ouma, J.H. \& Beier, J.C. (1993) Evaluation of light traps for sampling anopheline mosquitoes in Kilifi, Kenya. Journal of the American Mosquito Control Association 9, 260-263.

Mogi, M., Miyagi, I., Toma, T., Hasan, M., Abadi, K. \& Syafruddin (1995) Age structure of Anopheles subpictus (Diptera: Culicidae) collected by a light trap in Halmahela, Indonesia. Southeast Asian Journal of Tropical Medicine and Public Health 26, 760-766.

Nedelman, J.A. (1983) A negative binomial model for sampling mosquitoes in a malaria survey. Biometrics 39, 1009-1020.

Parsons, R.E., Dondero, T.J. \& Cheong, W.H. (1974) Comparison of CDC miniature light traps and human biting collections for mosquito catches during malaria vector surveys in peninsular Malaysia. Mosquito News 34 211-213

Rubio-Palis, Y. (1996) Evaluation of light traps combined with carbon dioxide and 1-octen-3-ol to collect anophelines in Venezuela. Journal of the American Mosquito Control Association 12, 91-96.

Rubio-Palis, Y. \& Curtis, C.F. (1992) Evaluation of different methods of catching anopheline mosquitoes in western Venezuela. Journal of the American Mosquito Control Association 8, 261-267.

Service, M.W. (1970) A battery-operated light trap for sampling mosquito populations. Bulletin of the World Health Organization 43, 635-641.

Shidrawi, G.R., Clarke, J.L. \& Boulzaguet, J.R. (1973) Assessment of the CDC miniature light trap for sampling malaria vectors in the Garki district, northern Nigeria. Unpublished WHO Technical Note no. 11, MPD/TN/73.1,44-49.

Smith, T. (1995) Proportionality between light trap catches and biting densities of malaria vectors. Journal of the American Mosquito Control Association 11, 377-378.

Sudia, W.D. \& Chamberlain, R.W. (1962) Battery-operated light trap, an improved model. Mosquito News 22, 126-129.

Taylor, L.R. (1984) Assessing and interpreting the spatial distributions of insect populations. Annual Review of Entomology 29, 321-357.

Thomas, A., Spiegelhalter, D.J. \& Gilks, W.R. (1992) BUGS: a program to perform Bayesian inference using Gibbs sampling. pp. 837-842 in Bernardo, J.M., Berger, J.O., David, A.P. \& Smith, A.F.M. (Eds) Bayesian statistics vol. 4. Oxford, Clarendon Press.

Venables, W.N. \& Ripley, B.D. (1994). Modern applied statistics with S-plus. New York: Springer-Verlag.

Wirtz, R.A., Zavala, F., Charoenvit, Y., Campbell G.H., Burkot, T.R., Schneider, I., Esser, K.M., Beaudoin, R.L. \& Andre, R.G. (1987) Comparative testing of monoclonal antibodies against Plasmodium falciparum sporozoites for ELISA development. Bulletin of the World Health Organization $\mathbf{6 5}$ 39-45.

Wirtz, R.A., Charoenvit, Y., Burkot, T.R., Esser, K.M., Beaudoin, R.L., Collins, W.E., Rosenberg, R. \& Andre, 
R.G. (1990) Evaluation of monoclonal antibodies against Plasmodium vivax sporozoites for ELISA development. Medical and Veterinary Entomology 5, 17-22.

Wirtz, R.A., Charoenvit, Y., Burkot, T.R. \& Rosenberg, R. (1992) Development and evaluation of an ELISA for Plasmodium vivax variant strain-VK247 sporozoites. Journal of Medical Entomology 29, 854-857.
World Health Organization (1975) Manual on practical entomology. Part II: Methods and techniques, WHO Offset Publications No. 13, Geneva, pp. 12-15.

(Accepted 20 March 2000)

(c) CAB International, 2000 